\title{
IMPORTÂNCIA DO FLUXO DE MASSA E DIFUSÃO NO SUPRIMENTO DE POTÁSSIO AO ALGODOEIRO COMO VARIÁVEL DE ÁGUA E POTÁSSIO NO SOLO(1)
}

\author{
R. H. OLIVEIRA ${ }^{(2)}$, C. A. ROSOLE $M^{(3)} \&$ R. M. TRIGUEIRO(2)
}

\begin{abstract}
RESUMO
A deficiência tardia de potássio na cultura do algodoei ro tem ocorrido com freqüência nas regiões do cerrado brasileiro. Um dos motivos poderia ser atribuído à baixa disponibilidade de água nessa época. Assim, procurou-se quantificar a contribui ção da difusão e do fluxo de massa no suprimento de $K$ às raízes do algodoeiro de acordo com a disponibilidade do nutriente e de água. Para tanto, realizou-se um experimento em vasos em casa de vegetação, utilizando amostras da camada arável $(0-20 \mathrm{~cm})$ de um Latossolo Vermelho típico, com $330 \mathrm{mg} \mathrm{kg}^{-1}$ de argila. 0 experimento constou de duas doses de potássio (15 e $\left.121 \mathrm{mg} \mathrm{dm}^{-3}\right)$, na forma de $\mathrm{KCl}$, e quatro conteúdos de água $(-0,03 ;-0,1 ;-0,5$ e $-1,0 \mathrm{MPa}$ ). As plantas foram colhidas aos 53 dias da emergência. A difusão foi o principal mecanismo de transporte de K no solo, variando de 72 a $96 \%$ do total absorvido pelo algodoeiro. A influência do conteúdo de água do solo sobre os mecanismos de transporte de $K$ foi mai or em solos com maior concentração deste nutriente, razão por que o fluxo de massa cresce em importância em solos mais secos.
\end{abstract}

Termos de indexação: Gossypium hi rsutum, nutrição mineral, solução do solo, transporte de $\mathrm{K}$.

\section{SUMMARY: IMPORTANCE OF MASS FLOW AND DIFFUSION ON THE POTASSIUM SUPPLY TO COTTON PLANTS AS AFFECTED BY SOIL WATER AND POTASSIUM}

Late season potassium $(K)$ deficiency has been observed quite frequently for cotton crops in the Brazilian cerrado region. One possiblereason for such a problem could be the low water availability at this period of the season. Thus, an experiment was conducted in

\footnotetext{
(1) Trabal ho apresentado no XXVIII Congresso Brasileiro de Ciência do Solo, Londrina (PR), de 01 a 06 de J ulho de 2001. Recebido para publicação em agosto de 2002 e aprovado em dezembro de 2003.

(2) Estudante de Pós-Graduação, Departamento de Produção Vegetal, Faculdade de Ciências Agronômicas - FCA/UNESP. Caixa Postal 237, CEP 18603-970 Botucatu (SP). E-mail: rosahonorato@fca.unesp.br

(3) Professor Titular do Departamento de Produção Vegetal, FCA/UNESP. Bosista do CNPq. E-mail: rosolem@fca.unesp.br
} 
order to quantify the relative contribution of mass flow and diffusion in supplying $K$ to cotton roots, as affected by soil water and $\mathrm{K}$ availability. The arable layer of a typic Red Latosol (Haplortox), with $630 \mathrm{mg} \mathrm{kg}^{-1}$ sand, $40 \mathrm{mg} \mathrm{kg}^{-1}$ silt, and $330 \mathrm{mg} \mathrm{kg}^{-1}$ clay was sampled and filled into $5 \mathrm{~L}$ pots. The treatments were two $\mathrm{K}$ rates ( 15 and $\left.121 \mathrm{mg} \mathrm{dm}^{-3}\right)$, appli ed as potassium chloride, and four levels of soil water $(-0.03,-0.1,-0.5$, and $-1.0 \mathrm{MPa})$. Two cotton plants were grown in each pot and harvested 53 days after plant emergence. Diffusion was the main transport mechanism of $K$ to cotton roots, contributing from 72 to $96 \%$ of thetotal uptake. Theeffect of soil water upon theK transport to theroot surfacewas greater in soils with a higher $\mathrm{K}$ concentration. That is the reason why mass flow is more important in dryer soils.

Index terms: Gossypium hirsutum, mineral nutrition, soil solution, $\mathrm{K}$ transport.

\section{INTRODUÇÃO}

A disponibilidade de nutrientes na rizosfera depende, dentre outros fatores, da concentração do nutriente na solução do solo e do transporte deste para a superfície radicular (Chen \& Gabelman, 2000). A concentração do nutriente na solução do solo é de importância primária para o suprimento deste nutriente para a raiz (Marshner, 1995).

Os nutrientes são transportados atéàs raízes por meio de três mecanismos: intercepção radicular, difusão e fluxo de massa. A intercepção radicular ocorre quando os nutrientes são inter ceptados pelas raízes durante o processo de crescimento; somente pequena percentagem do nutriente total requerido é suprida por este processo (Marshner, 1995). Entretanto, o mecanismo de intercepção radicular não tem sido considerado no transporte de nutrientes, pois, atualmente, não é aceita a possibilidade de trocas diretas entre as partículas do sol o e as raízes das plantas, havendo necessidade do meio líquido para que a absorção pelas raízes ocorra (Ruiz et al., 1999). A difusão é caracterizada pel o movimento térmico ao acaso de íons em direção à raiz, em virtude do gradiente de concentração gerado na superfície radicular (Barber, 1984), enquanto o fluxo de massa está associado ao gradiente de potencial hídrico provocado pela absorção de água pelas plantas (Silva et al., 1998).

O K é bastante móvel no solo e sua absorção é altamente seletiva (Chen \& Gabelman, 2000). Embora não sejam conhecidas as funções desse nutriente, sabe-se que eleativa mais de 60 enzimas. Suas principais funções estão ligadas a processos vitais, tais como: fotossíntese, transl ocação e balanço iônico (Marschner, 1995). A difusão é o principal mecanismo de transporte do K atéa raiz; contudo, o fluxo de massa pode ter contribuição significativa no processo quando a concentração de $K$ na sol ução do solo é el evada (Ruiz et al., 1999).

O algodoeiro é uma cultura bastante exigente e muito responsiva a $\mathrm{K}$ em termos de produtividadee qualidade de fibra, produzindo mais quando em solo de alta fertilidade, sob condições adequadas de umidade do solo, altas temperaturas e alta intensi dadeluminosa (Grespan \& Zancanaro, 1999). Apesar disso, éuma cultura que apresenta uma taxa diária de consumo hídrico relativamente baixa (cerca de 6,5 $\mathrm{mm} \mathrm{dia}^{-1}$, na fase de maior demanda transpiratória), mesmo em climas quentes e com suprimento adequado de água no solo. No caso de variedades precoces, o consumo hídrico durante o ciclo vegetativo não ultrapassa $450 \mathrm{~mm}$ (Azevedo et al., 1993). Em variedades modernas, no final do ciclo, é comum ocorrer deficiência de $K$. N ormalmente, o final do cicl o do algodoeiro coincide com épocas de menor pluviosidade e, portanto, de menos água disponível no solo. Como a maior parte do K étransportada atéà raiz por difusão, processo altamente dependente da água do solo, à medida quea planta desenvol ve e o sol o seca, diminui oteor do nutriente que pode ser difundido, justificando, possivelmente, a deficiência observada ao final do ciclo da planta.

O objetivo do presente trabalho foi avaliar a contribuição da difusão e do fluxo de massa no suprimento deK para a cultura do algodão de acordo com a água disponível e com a disponibilidade de K no solo.

\section{MATERIAL E MÉTODOS}

Realizou-se um experimento em condições de casa de vegetação, no Departamento de Produção Vegetal, FCA/U nesp, Botucatu, no período de outubro a dezembro de 2000 . O solo utilizado foi um Latossolo Vermelho distroférrico típico (EMBRAPA, 1999), com $630 \mathrm{mg} \mathrm{kg}^{-1}$ de areia, $40 \mathrm{mg} \mathrm{kg}^{-1}$ de silte e $330 \mathrm{mg} \mathrm{kg}^{-1}$ de argila, com as seguintes características de fertilidade, determinadas segundo indicações de Raij \& Quaggio (1983): $\mathrm{pH}$ em $\mathrm{CaCl}_{2}$ 0,01 $\mathrm{mol} \mathrm{L}^{-1}$ de 4,1; $18 \mathrm{~g} \mathrm{dm}^{-3}$ de M.O.; $1 \mathrm{mg} \mathrm{dm}^{-3} \mathrm{de}_{\text {resina; }} ; 5 \mathrm{mmol}_{c} \mathrm{dm}^{-3}$ $\mathrm{de} \mathrm{H}+\mathrm{Al} ; 4 \mathrm{mmol}_{\mathrm{C}} \mathrm{dm}^{-3}$ de $\mathrm{Ca}^{2+} ; 1 \mathrm{mmol}_{\mathrm{C}} \mathrm{dm}^{-3}$ de $\mathrm{Mg}^{2+} ; 0,1 \mathrm{mmol}_{\mathrm{c}} \mathrm{dm}^{3} \mathrm{de} \mathrm{K}^{+} ; 5 \mathrm{mmol}_{\mathrm{C}} \mathrm{dm}^{-3}$ de soma de bases; CTC $\mathrm{pH} 7,0$ de $80 \mathrm{mmol}_{\mathrm{c}} \mathrm{dm}^{-3}$ e $6 \%$ de V. Foi realizada calagem para elevação da 
saturação por bases para $60 \%$, seguida de incubação úmida (aproximadamente $80 \%$ da capacidade de retenção de água do solo) por 15 dias. Após este período, foi realizada a adubação com N $\left(50 \mathrm{mg} \mathrm{dm}^{-3}\right)$ e P (150 $\left.\mathrm{mg} \mathrm{dm}^{-3}\right)$, nas formas de sulfato de amônio e superfosfato simples. Nessa mesma época, foram aplicadas as doses de $\mathrm{K}$ que, juntamente com os conteúdos de água, constituíram os tratamentos.

O experimento foi instalado em esquema fatorial $2 \times 4$, com duas doses de $\mathrm{K}$ ( 15 e $121 \mathrm{mg} \mathrm{dm}^{-3}$ ), como cloreto de potássio, e quatro potenciais de água no solo (-0,03; - 0,$1 ;-0,5$ e -1,0 M Pa), correspondendo a 108, 92, 81 e $77 \mathrm{~g} \mathrm{~kg}^{-1}$ de água no solo, com quatro repetições, arranjadas em blocos casualizados. Utilizaram-se vasos de polietileno com capacidade para $5 \mathrm{~kg}$ de solo. Em duas repetições de cada tratamento, foram instaladas cápsulas porosas de porcelana para extração de solução do solo. As cápsulas tinham $100 \mathrm{~mm}$ de comprimento e $20 \mathrm{~mm}$ de diâmetro externo e foram instaladas, verticalmente, a partir do fundo do vaso.

Semeou-se o algodão (Gossypium hirsutum cv. Delta Opal), colocando-se cinco sementes por vaso. Dez dias após a emergência das plântulas, foi realizado o desbaste, deixando-se duas plantas por vaso, quando foram estabelecidos os conteúdos de água. O controle da umidade foi feito por meio de pesagens diárias e reposi ção da água evapotranspirada. $\mathrm{O}$ tempo para que cada potencial fosseatingido variou conforme a temperatura no período. Da semeadura até 18 dias após a emergência, a umidade do solo nos vasos foi corrigida a -0,03 MPa, a cada dois dias, quando os tratamentos passaram a ser considerados. Além dos vasos com plantas, foram preparados dois vasos, sem plantas, para cada conteúdo deágua, para monitorar a perda de água e estimar a evaporação.

A extração da solução do solo foi realizada em três épocas ao longo do experimento, em intervalos de 15 dias, sendo a primeira aos 33 dias da emergência das plantas. As coletas de solução do solo, entre 50 e $100 \mathrm{~mL}$, foram realizadas com auxílio de uma bomba a vácuo, na manhã seguinte à el evação da umidade do sol o a-0,03 MPa. A solução foi filtrada, acondicionada em frascos de vidros e armazenada sob refrigeração. Em cada coleta o K foi determinado por leitura direta em ICP (Induced Coupled Plasma).

As plantas foram colhidas aos 63 dias da emergência, divididas em parte aérea eraízes esecas em estufa de circulação forçada a $60^{\circ} \mathrm{C}$, onde permaneceram até atingir peso constante. Após a secagem, o material foi pesado, moído e submetido à análise para determinação dos teores de K, segundo Malavolta et al. (1997). Antes da secagem, foi tomada uma amostra de aproximadamente $30 \%$ do sistema radicular, para determinar o comprimento e o diâmetro, utilizando-se o software Winrhizo, versão 3.8.
Para cal cular a quantidade deágua transpirada, subtraiu-se a quantidade evaporada do volumetotal aplicado em cada vaso. O K absorvido, suprido por fluxo de massa, foi obtido, multiplicando-se a concentração do $\mathrm{K}$ na solução do solo (média entre duas extrações sucessivas) pelo volume de água transpirado no período. A difusão foi calculada subtraindo-se o valor correspondente do $\mathrm{K}$ transportado por fluxo de massa do $\mathrm{K}$ total acumulado na planta (Becker \& Maures, 1986; Barber, 1894).

Os resultados foram submetidos à análise de variância, utilizando o teste de Tukey $(p<0,05)$. Quando apropriado, foram ajustadas curvas de regressão, escol hendo-se a equação matemática de melhor ajuste aos dados obtidos.

\section{RESULTADOS E DISCUSSÃO}

E mbora Rossetto et al . (1995) tenham observado maior volume deraízes de soja em sol os mais pobres em $K$, no presente trabalho, não houve efeito significativo da disponibilidade do nutriente no comprimento ou diâmetro radicular. Os conteúdos deágua também nãotiveram efeito no comprimento (média de 27,1 m por planta) ou diâmetro (média de $0,48 \mathrm{~mm}$ ) radicular do al godoeiro.

Não foi observado efeito $(p<0,05)$ dos conteúdos de água no solo sobre a produção de matéria seca. Entretanto, a matéria seca das plantas que receberam maior dose de $\mathrm{K}$ (10,5 g em média) foi maior que as com menor disponibilidade do nutriente (8,9 g em média).

Não houve modificação dos teores de $\mathrm{K}$ na matéria seca da parte aérea do algodoeiro com a redução na disponibilidade deágua no solo. Na dose de $121 \mathrm{mg} \mathrm{dm}^{-3}$, o teor médio foi de $26 \mathrm{~g} \mathrm{~kg}^{-1}$, enquanto no menor nível deK oteor médio foi menor $\left(14 \mathrm{~g} \mathrm{~kg}^{-1}\right)$. Os teores de $\mathrm{K}$ na matéria seca de raiz (Figura 1a) aumentaram até - 0,5 MPa com $121 \mathrm{mg} \mathrm{dm}{ }^{3}$ de $\mathrm{K}$ no solo e até $-1,0 \mathrm{MPa}$ com $15 \mathrm{mg} \mathrm{dm}^{-3}$ de $\mathrm{K}$ no solo. Na presença de maior dose de K, os teores do nutriente sempre foram maiores.

Os conteúdos de água não influenciaram o conteúdo deK na parteaérea do al godoei ro, embora o conteúdo na dose de $121 \mathrm{mg} \mathrm{dm}^{-3}$ (116,5 mg planta-1) tenha sido maior que aquele obtido na dose de $15 \mathrm{mg} \mathrm{dm}^{-3}$ (68 $\mathrm{mg} \mathrm{planta}^{-1}$ ), o queé justificado pelo aumento na concentração do nutriente na solução do solo. É possível que este comportamento seja devido à baixa taxa de consumo hídrico do al godoeiro, mesmo com suprimento adequado de água no solo, conforme sugeriram Azevedo et al . (1993). A menor dose de K aplicada no sol o proporcionou acúmulo de $\mathrm{K}$ na raiz com a redução na disponibilidade de água (Figura 1b), evidenciando, provavel mente, um efeito 



Figura 1. Concentração de potássio na matéria seca da raiz (a) e acúmulo de potássio na raiz do algodão (b), considerando o conteúdo de água no solo e a aplicação de potássio.

de concentração, uma vez que não houve efeito na produção de matéria seca.

A concentração de $K$ na solução do solo caiu expressivamente a cada extração sucessiva, para todos os conteúdos de água (Figura 2ab). O fenômeno deveu-se, pel omenos em parte, à demanda inicial da cultura pelo nutriente. Outra explicação seria que o K não permanece durante muito tempo em solução, tendendo a ficar adsorvido nos colói des do solo e nos resíduos de cargas da parede e na membrana celular das plantas. De acordo com Rosolem et al. (1993), as trocas entre K trocável e K solução são rápidas, de maneira que o K trocável é prontamente disponível às plantas. Desta forma, para a dose de $15 \mathrm{mg} \mathrm{dm}^{-3}$, observou-se um esgotamento deK na solução do sol o antecipadamente ao esgotamento que ocorreu na maior dose de K. Além disso, os tratamentos com menores potenciais de água (-0,5 e -1,0 MPa) apresentaram maiores teores do nutriente. Assim, o efeito da água na concentração de $K$ na solução do solo é mais importante em situação de alta disponibilidade do nutriente. $\mathrm{O}$ método utilizado no trabal ho leva a um decréscimo momentâneo no K solução que, no entanto, representa pequena parte do K disponível. Considerando que, nos cál culos, foi considerada a média entre duas amostragens e consi derando ainda que a quantidade de $\mathrm{K}$ extraído do solo representa menos de 3\% do total absorvido pela planta, este pode ser considerado um erro desprezível.

Quanto aos mecanismos de suprimento de K às raízes, não foi observado efeito dos conteúdos deágua na menor dose de K (Figura 3ab). Contudo, com maior disponibilidade do nutriente, houve um decréscimo no suprimento por difusão (Figura 3a), à medida que o solo estava mais seco, acompanhado de um aumento da contribuição do fluxo de massa (Figura $3 b$ ). I sso deve ter ocorrido em virtude do aumento da concentração de K na solução do solo (Figura 2ab). Este é um aspecto importante que atesta não só a relevância da concentração do nutriente na solução do solo para o suprimento à raiz (Marshner, 1995), mas também sua dependência do potencial matricial. A baixa disponibilidade de água no solo pode afetar a absorção de $\mathrm{K}$ em

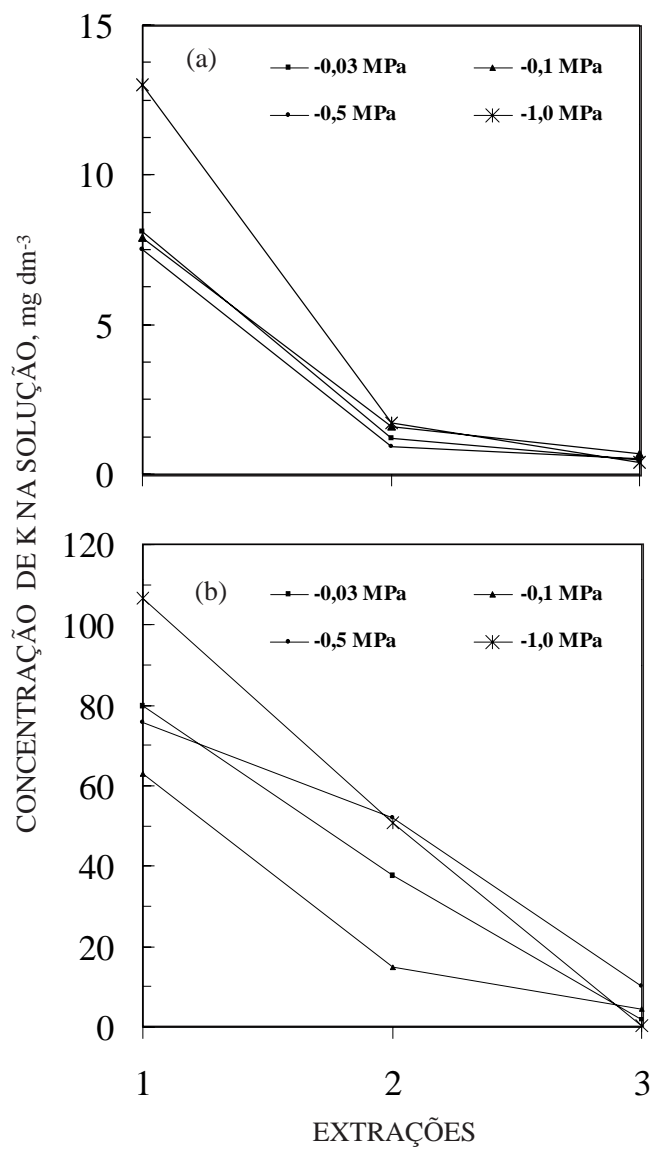

Figura 2. Esgotamento de potássio na solução do solo no tratamento com $15 \mathrm{mg} \mathrm{dm}^{-3}$ (a) e $121 \mathrm{~g} \mathrm{dm}^{-3}$ (b) de K, considerando a disponibilidade de água no solo e a aplicação de potássio. 
decorrência da restrição do crescimento radicular e da capacidade de absorção da raiz, assim como da menor mobilidade do K no meio (Seiffert et al., 1995). Ademais, a taxa de crescimento radicular étida como o fator que mais influencia a absorção de K (Silberbush \& Barber, 1983), de modo que, no caso derestrição ao crescimento radicular, a contribuição do fluxo de massa para osuprimento deK passaria a ser bastanterepresentativa. Entretanto, no presente experimento, não foi observado efeito na morfologia radicular, levando à inferência de quea modificação na importância dos mecanismos de contato tenha sido uma conseqüência de modificações na cinética de absorção (E pstein, 1976).

É importante lembrar que, no período do experimento, a exigência da planta em água foi relativamente baixa (Grimes \& EI-Zick, 1990). Como conseqüência deste fato, não foi observada diferença na quantidade de água transpirada, seja em função da disponibilidade de $K$, seja de água (média de $2.730 \mathrm{~cm}^{3}$ por planta).

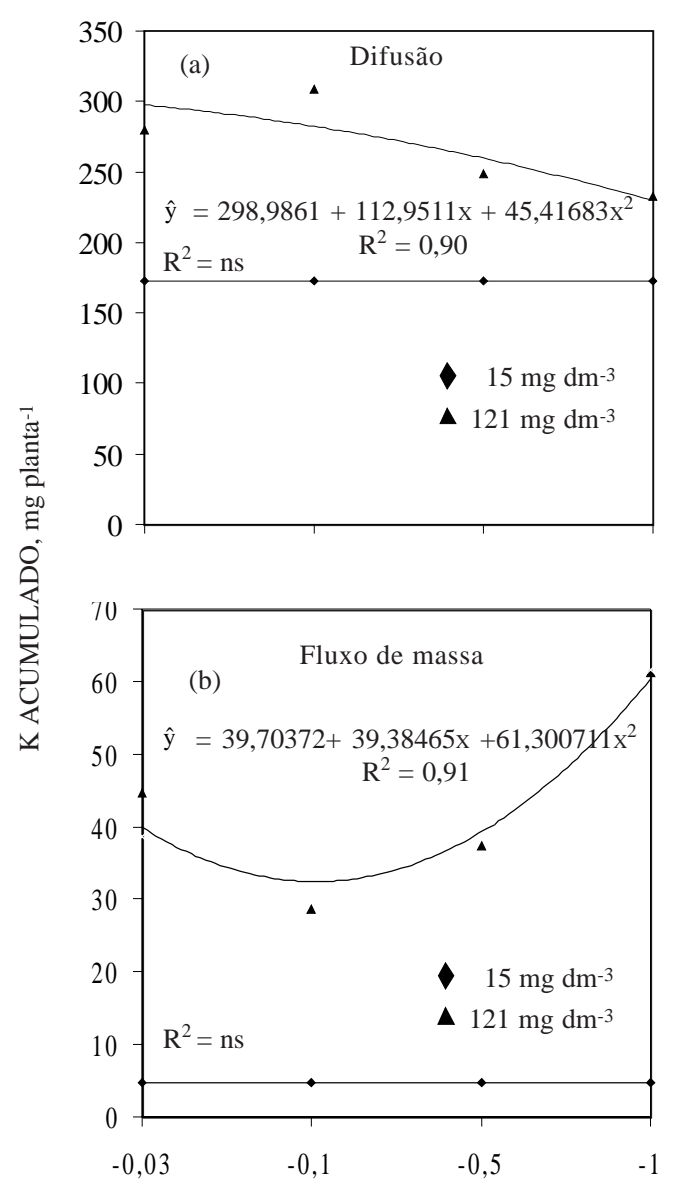

POTENCIAL DE ÁGUA NO SOLO (MPA)

Figura 3. Conteúdo de potássio em plantas em decorrência do transporte por difusão (a) e por fluxo de massa (b), considerando a disponibilidade de água no solo e a aplicação de potássio.
Considerando a equação da difusão de K no solo, a adição do nutriente el eva sua concentração na solução, aumentando o gradiente de concentração $(\delta C / \delta x$, em que $C$ é concentração e $x$ distância), aumentando a difusão (Ching \& Barber, 1979). Por outro lado, a diminuição da água disponível levaria a uma diminuição na difusão. Maior conteúdo de água no sol o favorecea difusão pel o seu efeito direto sobre o coeficiente de difusão e por reduzir a tortuosidade do caminho difusivo, aumentando, também, o valor do coeficiente de difusão (Barber, 1984). U ma redução do conteúdo de água no sol o de $0,35 \mathrm{~cm}^{3} \mathrm{~cm}^{-3}$ para $0,3 \mathrm{~cm}^{3} \mathrm{~cm}^{-3} \mathrm{faz}$ a difusão decrescer de $1,45 \cdot 10^{-11}$ para $8,65 \cdot 10^{-12}$ moles de $\mathrm{K} \mathrm{cm}^{-2} \mathrm{~s}^{-2}$ (Hylander et al., 1999).

Outro fato a ser considerado é a distância média entre as raízes. Aceita-se que, quando a distância média entreas raízes émenor que $4 \mathrm{~mm}$, podehaver competição por K (Yamaguchi \& Tanaka, 1990). No presente caso, a distância média entre raízes, calculada como $1 /(\pi L)^{1 / 2}$, sendo $L$ densidade de comprimento, em cm cm $\mathrm{cm}^{-3}$ (Baldwin et al., 1973), foi sempre menor que $4,8 \mathrm{~mm}$, permitindo a inferência deque, no caso, ocorreu competição peloK difundido entre raízes vizinhas. A proporção do K transportado para as raízes por difusão decresce com o esgotamento do nutriente na rizosfera (Hylander et al., 1999), de modo que uma grande concentração radicular leva a esgotamento mais rápido do $\mathrm{K}$ solo na vizinhança das raízes (Seiffert et al., 1995). Outros trabalhos têm abordado a importância da taxa de crescimento da raiz, do comprimento radicular e da área superficial na absorção deK pelas plantas (Chen \& Gabel man, 2000), mas, no presente caso, não houve modificação dessas características.

Assim, com a menor dose de $\mathrm{K}$, a disponibilidade era tão baixa (Figura 2a) quelimitou a absorção em grau maior que a disponibilidade de água. Quando a umi dade não foi limitante, sem grande modificação na taxa transpiratória, a maior concentração de K na solução do solo mais seco proporcionou maior transporte do nutriente às raízes, fato também observado por Hylander et al. (1999).

Essa inferência foi confirmada pelos dados apresentados na figura 4. Observa-se quea difusão contribuiu, em média, com $96 \%$ do suprimento de K para as raízes deal godão, contra aproximadamente $3,5 \%$ do fluxo de massa, quando havia menos $\mathrm{K}$ no solo (Figura 4a). No caso de maior teor de $K$ disponível (Figura 4b), a média da difusão caiu para cerca de $80 \%$, e a contribuição de fluxo de massa passou de 3,5 para $19 \%$. Por outro lado, a menor disponi bilidade deágua, al iada à grande concentração radicular, levou ao menor transporte do $\mathrm{K}$ por difusão. Os resultados para fluxo de massa estão próximos aos obtidos por Rosolem et al. (2001), quando observaram contribuição de $23,7 \%$ com uma dose de $60 \mathrm{mg} \mathrm{kg}^{-1}$ de $\mathrm{K}$.

Barber (1984), estudando plantas de milho, observou quea difusão, em solo rico em K, contribuiu 
$\square$ Difusão $\quad \square$ Fluxo de massa

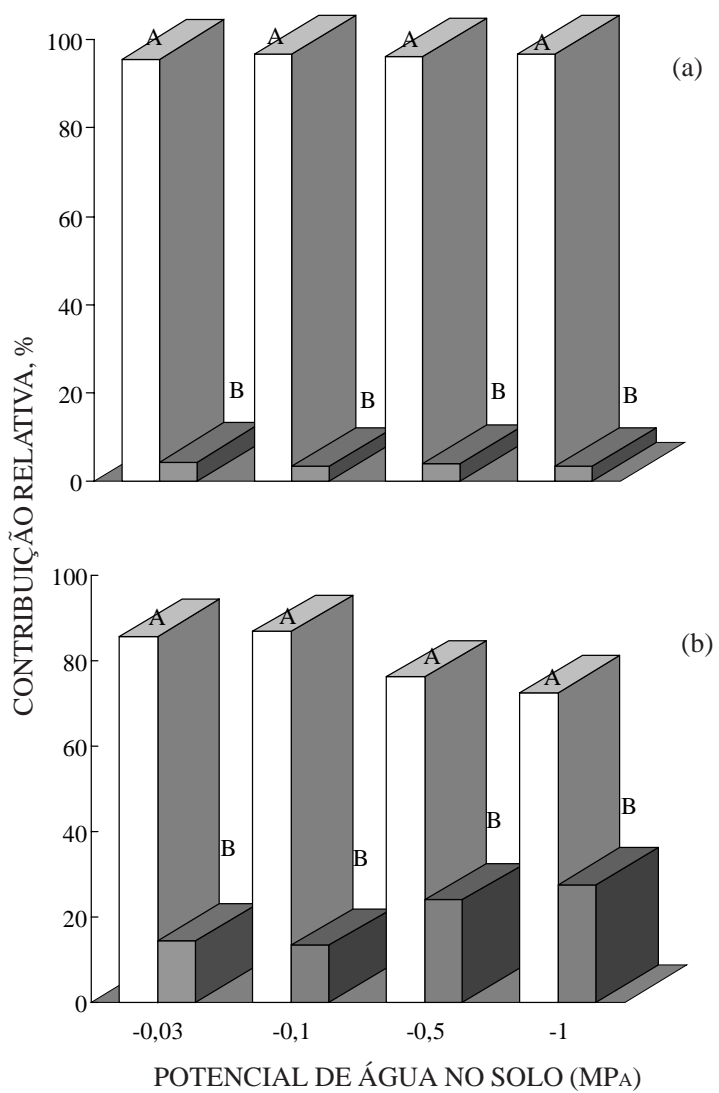

Figura 4. Contribuição percentual da difusão e do fluxo de massa na absorção de potássio, considerando a disponi bilidade de água no solo e a aplicação do potássio (a: $15 \mathrm{mg} \mathrm{dm}^{-3}$ e b: $121 \mathrm{mg} \mathrm{dm}^{-3}$ ). Barras com a mesma letra não diferem entre si pelo teste de Tukey a $5 \%$.

em média com cerca de $80 \%$ da quantidade do nutriente absorvido pela planta, o que mostra que, embora o algodão e o milho tenham sistemas radiculares muito diferentes, isso não altera a importância relativa dos mecanismos de transporte do $\mathrm{K}$ no solo. Em ambas as concentrações de $\mathrm{K}$ na solução do solo, a difusão foi o processo dominante no suprimento deste nutriente para as raízes, resultados condizentes com os obtidos em outros trabalhos (Ruiz et al., 1999).

\section{CONCLUSÕES}

1. A difusão foi o principal mecanismo de transporte do K no solo, contribuindo com 72 a $96 \%$ do total absorvido pel o al godoeiro.

2. A maior disponibilidade deK na solução do solo proporcionou aumento da contribuição da difusão para o transporte de $\mathrm{K}$ às raízes do algodoeiro.
3. A influência da água do solo nos mecanismos de transporte doK foi maior em solos que continham mai ores concentrações deK em sol ução, tendo o fluxo de massa crescido em importância no caso de solo mais seco.

\section{LITE RATURA CITADA}

AZEVEDO, P.V.; RAO T.V.R.; AMORIM NETO, M.S.; PEREIRA, J.R.C.; ESPÍDOLA SOBRINHO, J \& \& MACIEL, G.F. Necessidades hídricas da cultura do algodoeiro. Pesq. Agropec. Bras., 28:863-870, 1993.

BALDWIN, J.P.; NYE, P.H. \& TINKER, P.B. U ptake of solutes by multiple root and systems from soil. III. A model for calculating the solute systems devel oping in a finite volume of soil. Plant Soil, 38:621-35, 1973.

BARBER, S.A. Soil nutrient biovailability: a mechanistic approach. New York, J ohn \& Wiley, 1984. 398p.

BECKER, M.\& MAURER, E.J . Morfologia deraízes, suprimento einfluxo de potássio em plantas de milho. R. Bras. Ci. Solo, 10:259-263, 1986.

CHEN, J \& \& GABELMAN, W.H. Morphological and physiological characteristics of tomato roots associated with potassiumacquisition efficiency. Sci. Hortic., 83:213-225, 2000.

CHING, P.C. \& BARBER, S.A. Evaluation of temperature effects on potassium uptake by corn. Agron. J ., 71:1040-1044, 1979.

EMPRESA BRASILEIRA DE PESQUISA AGROPECUÁRIA EMBRAPA. Centro de Pesquisa de Solos (Rio de J aneiro, RJ ). Sistema Brasileiro de Classificação de Solos. Rio de J aneiro, 1999. 412p.

EPSTEIN, E. Kinetics of ion transport and the carrier concept. In: LÜTTGE, U. \& PITMAN, M.G., eds. Transport in plants. Encyclopedia of plant physiology. New York, Berlin, Springer-Verlag, 1976. p.70-94.

GRESPAN, S.L. \& ZANCANARO, L. Nutrição e adubação do algodoeiro no Mato Grosso. Rondonópol is, FMT/EMBRAPA, 1999. p.95 (Boletim, 3)

GRIMES, D.W. \& EL-ZICK, K.M. Cotton. In: STEWART, B.A. $\&$ NIELSEN, D.R., eds. Irrigation of agricultural crops. Madison, American Society of Agronomy, 1990. p.712-773.

HYLANDER, L.D.; AE, N.; HATTA, T. \& SUGIYAMA, M. Exploitation of $K$ near roots of cotton, maize, upland rice and soybean grown in an Ultisol. Plant Soil, 208:33-41, 1999.

MALAVOLTA, E.; VITTI, G.C. \& OLIVEIRA, S.A. Avaliação do estado nutricional das plantas: princípios e aplicações. Piracicaba, POTAFOS, 1997. 319p.

MARSCHNER, H. Mineral nutrition of higher plants. 2.ed. Londres, Academic Press, 1995. p.347-364.

RAIJ , B. van \& QUAGGIO, J.A. Métodos de análises de solo para fins de fertilidade. Campinas, Instituto Agronômico de Campinas, 1983. 31p. (Boletim Técnico, 81)

ROSOLEM, C.A.; BESSA, A.M. \& PEREIRA, H.F.M. Dinâmica do potássio no solo e nutrição potássica da soja. Pesq. Agropec. Bras., 28:1045-1054, 1993. 
ROSOLEM, C.A.; ESTEVES, J .A.F. \& SILVA, R.H. Significance of mass flow and diffusion in supplying $K$ to cotton roots as affected by limiting and $K$ rates. In: INTERNATIONAL SYMPOSYUM ON PLANT-SOIL INTERATIONS AT LOW pH, 5., 2001, Abstracts. South Africa, Kualazulu-Natal, Departament of Agriculture and Environmental Affairs, 2001. p.48.

ROSSETTO, C.A.V.; FERNANDES, D.M.; ISHIMURA, I. \& ROSOLEM, C.A. Diferentes respostas de cultivares de soja ao potássio. Pesq. Agropec. Bras., 38:1225-1231, 1995.

RUIZ, H.A.; MIRANDA, J . \& CONCEIÇÃO, J .C.S. Contribuição dos mecanismos de fluxo de massa e de difusão para o suprimento de $\mathrm{K}, \mathrm{Ca}, \mathrm{Mg}$ às plantas de arroz. R. Bras. Ci. Solo, 23:1015-1018, 1999.
SEIFFERT, S.; KASELOWSKY, J .; JUNGK, A. \& CLAASSEN, N. Observed and calculated potassium uptake by maize as affected by soil water content and bulk density. Agron. J ., 87:1070-1077, 1995.

SILBERBUSH, M. \& BARBER, S.A. Sensitivity analysis of parameters used in simulating uptake with a mechanistic mathematic model. Agron. J ., 75:851-854, 1983.

SILVA, D.J .; ALVAREZ, V.H. \& RUIZ, H.A. Fluxo de massa e difusão de enxofre para raízes de milho em solos ácidos de Minas Gerais. R. Bras. Ci. Solo, 22:109-114, 1998.

YAMAGUCHI, J . \& TANAKA, A. Quantitative observation on the root systems of various crops growing in the field. Soil Sci. Plant Nutr., 36:483-493, 1990. 